\title{
Cavity characteristics of selectively oxidized vertical-cavity lasers
}

\author{
Kent D. Choquette, ${ }^{\text {a) }}$ K. L. Lear, R. P. Schneider, Jr., and K. M. Geib \\ Photonics Research Department, Sandia National Laboratories, Albuquerque, New Mexico 87185
}

(Received 27 January 1995; accepted for publication 25 April 1995)

\begin{abstract}
We show that a buried oxide layer forming a current aperture in an all epitaxial vertical-cavity surface emitting laser has a profound influence on the optical and electrical characteristics of the device. The lateral index variation formed around the oxide current aperture leads to a shift in the cavity resonance wavelength. The resonance wavelength under the oxide layer can thus be manipulated, independent of the as-grown cavity resonance, by adjusting the oxide layer thickness and its placement relative to the active region. In addition, the electrical confinement afforded by the oxide layer enables record low threshold current densities and threshold voltages in these lasers. (C) 1995 American Institute of Physics.
\end{abstract}

Vertical-cavity surface emitting lasers (VCSELs) are of interest from both scientific and technological points of view. The distributed Bragg reflector (DBR) mirrors used to provide longitudinal optical confinement have enabled the study of microcavity optical phenomena and are critical to VCSEL performance. In the transverse direction, optical and electrical confinement is commonly achieved using etched air-post index-guided structures, ${ }^{1}$ or ion implanted "gain-guided" devices. ${ }^{2}$ Recently, oxides formed from AlGaAs layers have been implemented into both edge emitting ${ }^{3}$ and verticalcavity laser diodes. ${ }^{4-7}$ We report optical and electrical properties of VCSELs fabricated using selective lateral oxidation to produce a buried oxide layer which forms a current aperture within an all semiconductor DBR mirror. We show that the buried oxide layer has profound implications, leading to new optical phenomena in the cavity as well as significant advances in VCSEL performance.

The selectively oxidized VCSEL structure ${ }^{6}$ is shown in Fig. 1. VCSEL diodes employing an oxide layer under a hybrid top dielectric DBR mirror have also been reported. ${ }^{4,5}$ The VCSELs described herein are grown by metalorganic vapor phase epitaxy and employ three InGaAs quantum wells designed to emit near $980 \mathrm{~nm}$ with parabolically graded mirror interfaces in the epitaxial DBRs. ${ }^{8}$ The high oxidation selectivity of AlGaAs alloys ${ }^{6}$ is exploited to form a single buried oxide layer between the active region and the top DBR as depicted in Fig. 1. Square mesas are reactive ion etched to expose the edge of the quarter-wavelength thick $\mathrm{AlGaAs}$ layer for lateral oxidation done at $425-450{ }^{\circ} \mathrm{C}$ in water vapor. The $\mathrm{AlGaAs}$ layer is partially oxidized laterally into the interior of the mesa to form a current aperture. The oxide current aperture thus minimizes current spreading and eliminates sidewall nonradiative recombination or implantation damage present in air-post or implanted structures, respectively. The smaller refractive index of the oxide layer ${ }^{9}$ also induces index-guided optical confinement, but in a planar VCSEL configuration amenable to efficient current flow and heat extraction. Greater optical and electrical confinement is achieved by employing current aperture oxide layers on both sides of the active region. Finally contrary to other reports, ${ }^{5}$ our oxidized VCSEL structure appears to be robust:

${ }^{a)}$ Electronic mail: kchoqu@sonnet.sandia.gov for example, a laser biased to produce $1 \mathrm{~mW}$ of $\mathrm{cw}$ output has shown continuous operation for $>600 \mathrm{~h}$.

One novel optical effect arising from the buried oxide layer is illustrated in Fig. 2. Because the refractive index is reduced from 3.0 for the original $\mathrm{AlGaAs}$ layer to $\approx 1.6$ for the oxidized layer, in addition to index-guided confinement, the longitudinal cavity resonance is also strongly modified under the oxide layer relative to the as-grown cavity resonance. Plotted in Fig. 2 is the calculated reflectance of two cavities, one with the top DBR mirror containing an oxide layer and the other with an unoxidized as-grown layer. Notice that with the oxide layer, the cavity resonance is blueshifted by $\approx 17 \mathrm{~nm}$ relative to the unoxidized cavity resonance wavelength. In addition, the reflectivity across the mirror stopband is increased for the DBR containing the oxide layer as compared to the as-grown DBR (not shown). Both of these attributes arise from the significantly smaller refractive index of the oxide layer, and lead to unique lasing characteristics.

Superimposed on Fig. 2 is the observed lasing spectrum from a VCSEL operating well above threshold which exhibits two simultaneous lasing emission separated by $17 \mathrm{~nm}$. This particular VCSEL has a blue-shifted offset in its peak laser gain relative to the (as-grown) cavity resonance wavelength. At threshold we observe lasing around the perimeter of the current aperture, which corresponds to the shorter wavelength emission in Fig. 2. At a higher injection current

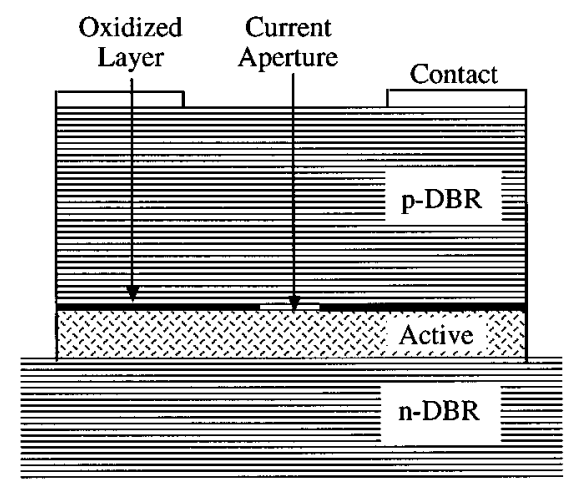

FIG. 1. Sketch of a selectively oxidized VCSEL showing the oxidized layer and the current aperture which is formed. 


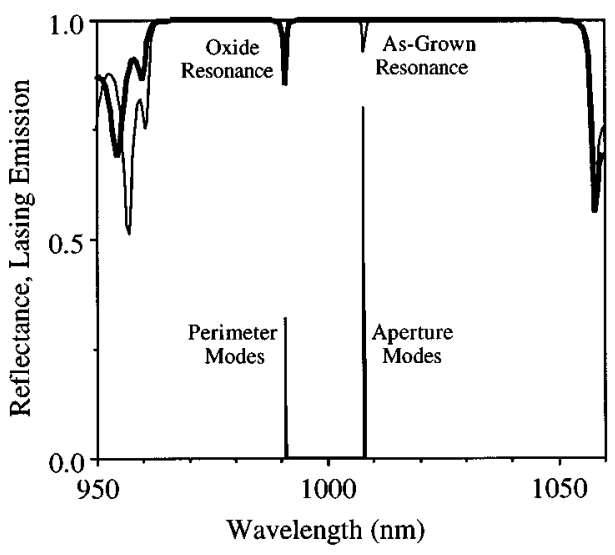

FIG. 2. The calculated cavity reflectance from the as-grown (unoxidized) mirror, and a mirror containing an oxidized layer; superimposed is the observed lasing emission above threshold.

the region inside the aperture also begins to lase, and a new lasing spectral peak is observed at the expected cavity resonance wavelength. Hence, due to the higher laser gain at the blue-shifted resonance and the higher reflectivity under the oxidized layer, "oxide modes" arise first at threshold around the perimeter of the current aperture where the carriers diffuse into the quantum wells under the oxide layer. As the laser gain red-shifts with increasing current injection, the gain comes into alignment with the as-grown cavity resonance, leading to simultaneous lasing "aperture modes." In regions of the wafer where the laser gain and as-grown resonance are near alignment, we typically only observe the expected aperture modes.

In Fig. 3 we show the calculated wavelength shift of the oxide cavity resonance relative to the as-grown cavity resonance using a single buried oxide layer. The vertical lines in Fig. 3 denote odd multiples of a quarter wavelength thickness of the unoxidized AlGaAs layer, the appropriate thicknesses for retaining the correct phase relationship in the un-

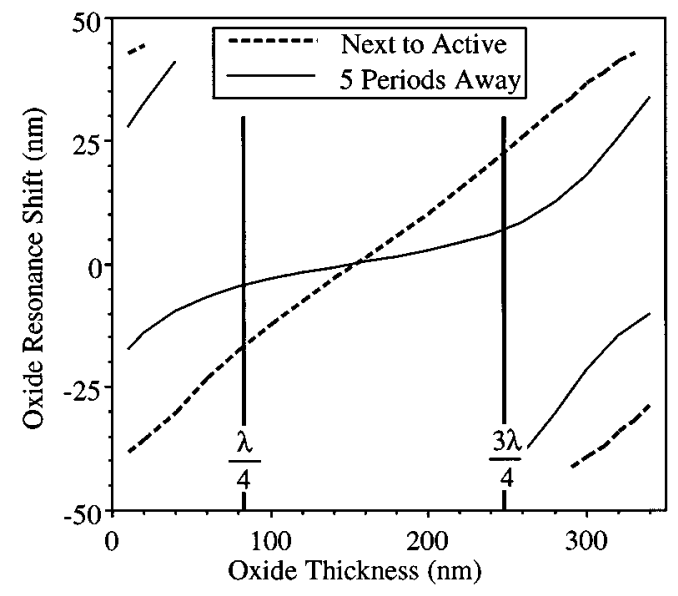

FIG. 3. The calculated shift of the oxide cavity resonance wavelength versus oxide thickness, relative to the as-grown cavity resonance wavelength. The solid curve corresponds to the oxide layer directly above the active region, while the broken curve corresponds to the oxide layer being placed 5 periods into the DBR mirror away from the active region.

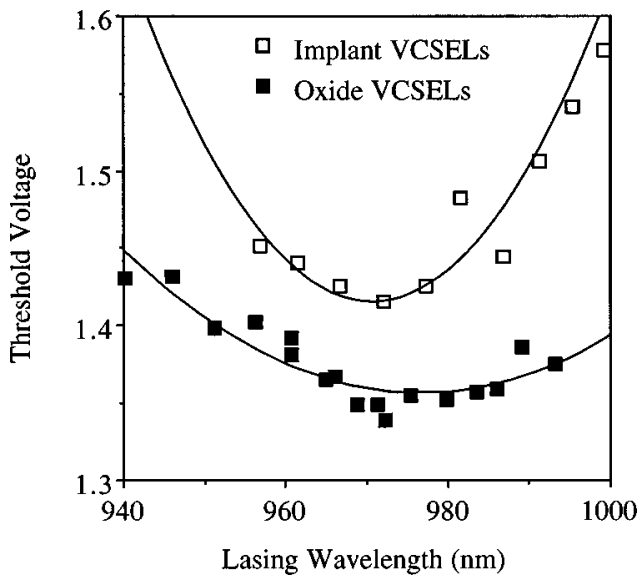

FIG. 4. Threshold voltage versus threshold lasing wavelength (at room temperature) for selectively oxidized and implanted VCSELs fabricated from the same wafer.

oxidized portion of the DBR. Notice that both blue- and red-shifted oxide cavity resonance wavelengths can be achieved by varying the thickness of the oxide layer. The magnitude of the shift can be reduced by inserting the oxide layer in the DBR mirror further away from the active region as also seen in Fig. 3. We find that multiple oxide resonances or no resonances can be found within the as-grown mirror stopband through appropriate design of the oxide layers. Independent control of the oxide cavity resonance may prove valuable to manipulate the optical cavity surrounding the VCSEL, for example to inhibit lasing and/or spontaneous emission.

The current aperture formed in the buried oxide layer provides enhanced electrical confinement as illustrated in Figs. 4 and 5. These figures compare the threshold voltage, $V_{\text {th }}$, and the threshold current density, $J_{\text {th }}$, of oxidized and implanted VCSELs fabricated from the same wafer. In these comparisons the oxidized VCSELs have aperture areas which vary from 700 to $1000 \mu \mathrm{m}^{2}$ across the sample, while the implanted VCSELs have a cross section area of 700 $\mu \mathrm{m}^{2}$. It is readily apparent from Figs. 4 and 5 that the oxidized VCSELs exhibit significantly lower $V_{\text {th }}$ and $J_{\text {th }}$ : the minimum values of $1.33 \mathrm{~V}$ (Ref. 6) and $90 \mathrm{~A} / \mathrm{cm}^{2}$ per quantum well, respectively, are the lowest values reported to date for VCSELs. Note from Fig. 1 that current flow is unimpeded through the DBR above the oxide aperture; thus the lateral and vertical resistance is reduced for the oxide VCSELs, as compared to implanted lasers. ${ }^{10}$ Therefore for the oxide VCSELs, the low $V_{\text {th }}$ arises from the low DBR series resistance ${ }^{8}$ and the efficient current injection. For these broad area lasers, the low $J_{\text {th }}$ primarily arises from strong electrical confinement (reduced current spreading) with an additional contribution from index-guiding (reduced diffraction loss).

The improved electrical properties of the oxide VCSEL have important performance consequences. First, the variation in $V_{\text {th }}$ and $J_{\text {th }}$ observed for differing laser gain/cavity resonance alignment in Figs. 4 and 5 is significantly reduced for the oxide VCSELs as manifest in the smaller curvature of 


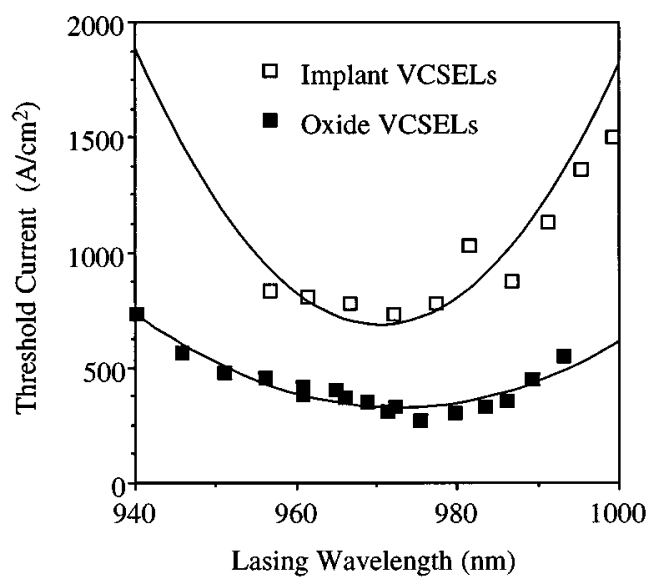

FIG. 5. Threshold current density versus threshold lasing wavelength (at room temperature) for selectively oxidized and implanted VCSELs fabricated from the same wafer.

their fitted curves in the figures. The lower electrical power requirements of the oxide VCSELs imply less heat generation and associated thermally-induced shifts in the laser gain and resonance wavelength; reduced gain/cavity resonance spectral mismatch produces a smaller variation in $V_{\text {th }}$ and $J_{\text {th }}$. This diminished variation is important to minimize the effects of growth and fabrication nonuniformities in VCSEL arrays. Secondly, the superior electrical and optical confinement will enable fabrication of small area devices producing ultra-low threshold currents. ${ }^{4,5,11}$ Finally and perhaps most importantly, the combination of enhanced electrical confinement and the inherent index-guiding in these selectively oxi- dized VCSELs has led to power conversion efficiencies of greater than $50 \%$, a significant advance in VCSEL performance. ${ }^{7}$

In summary, our selectively oxidized all epitaxial VCSEL structure allows the manipulation of the off-axis cavity resonance independent of the as-grown cavity resonance. This effect will enable tailoring the lateral optical cavity characteristics, perhaps leading to new microcavity phenomena. In addition, the oxidized VCSEL structure is shown to inherently possess enhanced electrical and optical confinement, which has led to notable advances in VCSEL performance. This work is supported by the United States Department of Energy under Contract No. DE-AC04-94AL85000.

${ }^{1}$ A. Scherer, J. L. Jewell, Y. H. Lee, J. P. Harbison, and L. T. Florez, Appl. Phys. Lett. 55, 2724 (1989).

${ }^{2}$ K. Tai, R. J. Fischer, K. W. Wang, S. N. G. Chu, and A. Y. Cho, Electron. Lett. 25, 1644 (1989).

${ }^{3}$ J. M. Dallesasse and N. Holonyak, Jr., Appl. Phys. Lett. 58, 394 (1991).

${ }^{4}$ D. L. Huffaker, D. G. Deppe, K. Kumar, and T. J. Rogers, Appl. Phys. Lett. 65, 97 (1994).

${ }^{5}$ D. L. Huffaker, J. Shin, and D. G. Deppe, Electron. Lett. 30, 1946 (1994).

${ }^{6}$ K. D. Choquette, R. P. Schneider, Jr., K. L. Lear, and K. M. Geib, Electron. Lett. 30, 2043 (1994).

${ }^{7}$ K. L. Lear, K. D. Choquette, R. P. Schneider, Jr., S. P. Kilcoyne, and K. M. Greib, Electron. Lett. 31, 208 (1995).

${ }^{8}$ K. L. Lear, R. P. Schneider, K. D. Choquette, S. P. Kilcoyne, J. J. Figiel, and J. C. Zolper, IEEE Photonics Technol. Lett. 6, 1053 (1994).

${ }^{9}$ F. A. Fish, S. J. Caracci, N. Holonyak, Jr., J. M. Dallesasse, K. C. Hsieh, M. J. Ries, S. C. Smith, and R. D. Burnham, Appl. Phys. Lett. 59, 1755 (1991).

${ }^{10}$ K. L. Lear, S. P. Kilcoyne, and S. A. Chalmers, IEEE Photonics Technol. Lett. 6, 778 (1994).

${ }^{11}$ K. L. Lear, K. D. Choquette, R. P. Schneider, Jr., and S. P. Kilcoyne 66, 2616 (1995). 